\title{
A NOTE ON THE BOUNDARY INTEGRAL EQUATION METHOD FOR THE SOLUTION OF SECOND ORDER ELLIPTIC EQUATIONS
}

\author{
D. L. CLEMENTS ${ }^{1}$, M. HASELGROVE ${ }^{1}$ AND D. M. BARNETT ${ }^{2}$
}

(Received 19 September 1983; revised 16 April 1984)

\begin{abstract}
The boundary integral equation method is obtained by expressing a solution to a particular partial differential equation in terms of an integral taken round the boundary of the region under consideration. Various methods exist for the numerical solution of this integral equation and the purpose of this note is to outline an improvement to one of these procedures.
\end{abstract}

\section{Introduction}

The boundary integral equation method is now a well established technique for the solution of boundary value problems governed by certain types of partial differential equations. The method is essentially obtained by expressing the solution to a particular boundary value problem in terms of an integral equation with the integral taken round the boundary of the region under consideration. This integral equation is then solved numerically by employing approximate quadrature formulas and then solving the resulting system of linear algebraic equations (see for example Jaswon and Symm [4] and Clements [1]). The aim of the present work is to introduce an improvement to this procedure for the class of problems which are governed by a system of second order, linear partial differential equations. This improvement is obtained by noting that in certain circumstances it is not necessary to employ approximate quadrature formulas since some

\footnotetext{
${ }^{1}$ Department of Applied Mathematics, University of Adelaide, S. A. 5000.

${ }^{2}$ Department of Materials Science and Engineering, Stanford University, Stanford, California 94305 , U.S.A.

( Copyright Australian Mathematical Society 1985, Serial-fee code 0334-2700/85
} 
of the relevant integrals may be integrated exactly. This leads to considerable simplification in the implementation of the boundary integral procedure and, in particular, makes it easier to write a computer program for the method.

\section{The boundary value problem}

Consider the system of elliptic partial differential equations

$$
a_{i j k l} \frac{\partial^{2} \phi_{k}}{\partial x_{j} \partial x_{l}}=0, \quad i, k=1,2, \ldots, N ; j, l=1,2,
$$

in which $\phi_{k}$ are functions of the dependent variables $x_{1}$ and $x_{2}$. In (2.1) the convention of summing over repeated Latin subscripts is employed. The constants $a_{i j k l}$ must satisfy certain symmetry and ellipticity conditions which are detailed in Clements and Rizzo [3]. The problem is to find a solution to (2.1) valid in a region $\mathscr{R}$ in $E^{2}$ with boundary $C$. On $C$ either the dependent variables $\phi_{k}$ are specified or the $P_{i}$ are specified where

$$
P_{i}=a_{i j k l} \frac{\partial \phi_{k}}{\partial x_{l}} n_{j}
$$

where $n_{j}$ is the unit (outer) normal to $\mathscr{R}$. If the $P_{i}$ are specified over all of $C$ we explictly require that

$$
\int_{c} P_{i} d s=0
$$

\section{The integral equation}

It has been shown by Clements and Rizzo [3] that an integral equation which solves the problem under consideration is

$$
\lambda \phi_{j}\left(\mathbf{x}_{0}\right)+\int_{c}\left[P_{i}(\mathbf{x}) \Phi_{i j}\left(\mathbf{x}, \mathbf{x}_{0}\right)-\Gamma_{i j}\left(\mathbf{x}, \mathbf{x}_{0}\right) \phi_{j}(\mathbf{x})\right] d s(\mathbf{x})=0
$$

where $\lambda=1$ if $\mathbf{x}_{0} \in \mathscr{R},\left[\mathbf{x}_{0}=(a, b)\right]$ and $0<\lambda<1$ if $x_{0} \in C$. If $C$ has a continuously turning tangent then $\lambda=\frac{1}{2}$. Also $\Phi_{i j}$ and $\Gamma_{i j}$ are given by

$$
\begin{gathered}
\Phi_{k m}=\frac{1}{2 \pi} \operatorname{Re}\left\{\sum_{\alpha} A_{k \alpha} N_{\alpha j} \log \left(z_{\alpha}-c_{\alpha}\right)\right\} d_{j m}, \\
\Gamma_{i m}=\frac{1}{2 \pi} \operatorname{Re}\left\{\sum_{\alpha} L_{i j \alpha} N_{\alpha p}\left(z_{\alpha}-c_{\alpha}\right)^{-1}\right\} n_{j} d_{p m},
\end{gathered}
$$


where $z_{\alpha}=x_{1}+\tau_{\alpha} x_{2}$ and $c_{\alpha}=a+\tau_{\alpha} b$, where $\tau_{\alpha}$ for $\alpha=1,2, \ldots, N$ are the roots with positive imaginary part of the polynomial in $\tau$.

$$
\left|a_{i 1 k 1}+a_{i 2 k 1} \tau+a_{i 1 k 2} \tau+a_{i 2 k 2} \tau^{2}\right|=0 .
$$

The $A_{k \alpha}$ occurring in (3.2) are the solutions of the system

$$
\left(a_{i 1 k 1}+a_{i 1 k 2} \tau_{\alpha}+a_{i 2 k 1} \tau_{\alpha}+a_{i 2 k 2} \tau_{\alpha}^{2}\right) A_{k \alpha}=0 .
$$

Also the $N_{\alpha j}, L_{i j \alpha}$ and $d_{r j}$ are defined by

$$
\begin{gathered}
\sum_{\alpha} A_{k \alpha} N_{\alpha j}=\delta_{k j}, \\
L_{i j \alpha}=\left(a_{i j k 1}+\tau_{\alpha} a_{i j k 2}\right) A_{k \alpha},
\end{gathered}
$$

and

$$
\delta_{i j}=-\frac{1}{2} i \sum_{\alpha}\left\{L_{i 2 \alpha} N_{\alpha r}-\bar{L}_{i 2 \alpha} \bar{N}_{\alpha r}\right\} d_{r j} .
$$

In general equation (3.1) does not provide a simple analytical solution to a boundary value problem of the type specified in Section 2. However it does form the basis for an effective procedure for solving particular problems numerically.

The numerical procedure involves solving the integral equation (3.1) for the unknown $\phi_{i}$ or $P_{i}$ on the boundary $C$. Once this has been done equation (3.1) then provides $\phi_{i}$ at all interior points.

Now it is clear that when (3.1) is used to obtain either $\phi_{i}$ or $P_{i}$ on $C$ the point $\mathbf{x}_{0}$ will be a point on $C$ so that the term $\Gamma_{i j}$ in the integrand gives rise to an improper integral. The method which is commonly used to circumvent this difficulty may be outlined as follows.

Substitution of the solution

$$
\phi_{i}=k_{i} \text { (constant) for } i=1,2, \ldots, N
$$

into (3.1) yields the set of equations

$$
k_{i} \int_{C} \Gamma_{i j}\left(\mathbf{x}, \mathbf{x}_{0}\right) d s(\mathbf{x})=\lambda k_{j}
$$

which has a solution for nonzero $k_{i}$ only if

$$
\int_{C} \Gamma_{i j}\left(\mathbf{x}, \mathbf{x}_{0}\right) d s(\mathbf{x})=\lambda \delta_{i j}
$$

where $\delta_{i j}$ is the Kronecker delta. Hence (3.1) can be written in the form

$$
\int_{C} P_{i}(\mathbf{x}) \Phi_{i j}\left(\mathbf{x}, \mathbf{x}_{0}\right) d x(\mathbf{x})=\int_{C}\left[\phi_{i}(\mathbf{x})-\phi_{i}\left(\mathbf{x}_{0}\right)\right] \Gamma_{i j}\left(\mathbf{x}, \mathbf{x}_{0}\right) d x(\mathbf{x}) .
$$

Unlike (3.1), equation (3.8) does not involve improper integrals if $\mathbf{x}_{0} \in C$ and hence it is a convenient method to employ for obtaining numerical values of 
either $\phi_{i}$ or $P_{i}$ on the boundary $C$. Once this has been done equation (3.1) then gives $\phi_{i}$ at all interior points of the region $\mathscr{R}$. Thus the solution of a boundary problem for equation (2.1) by this boundary integral procedure involves use of both (3.1) and (3.8) for numerical values. Furthermore the use of (3.8) to obtain the relevant boundary values is a reasonably complicated exercise. (See for example Clements and Jones [2].) The aim here is to show that there is no need to employ (3.8) since (3.1) can be effcctively used to obtain the requiired bounindary values and furthermore the procedure for obtaining these values is both simpler and more precise than the corresponding procedures which have been used on (3.8).

To obtain a numerical solution to (3.1) the boundary is discretised into $M$ segments $\left[\mathbf{q}_{1}, \mathbf{q}_{2}\right],\left[\mathbf{q}_{2}, \mathbf{q}_{3}\right], \ldots,\left[\mathbf{q}_{M}, \mathbf{q}_{1}\right]$ where the points $\mathbf{q}_{i}$ lie on $C$. It is assumed that $P_{i}$ and $\phi_{i}$ are constant on each segment, that is we set

$$
P_{i}(\mathbf{x})=P_{i j}, \quad \phi_{i}(\mathbf{x})=\phi_{i j} \quad \text { on }\left[\mathbf{q}_{j}, \mathbf{q}_{j+1}\right],
$$

where $P_{i j}$ and $\phi_{i j}$ are constants. By taking $\mathbf{x}_{0}$ to be the $M$ midpoints $\overline{\mathbf{q}}_{m}$ of each segment $\left[\mathbf{q}_{m}, \mathbf{q}_{m+1}\right]$ in turn we have a set of $M \times N$ equations in the $M \times N$ unknowns $P_{i j}$ or $\phi_{i j}$, which are the complimentary set to the values of $P$ and $\phi$ given on the boundary. The integral in (3.1) is now taken around the boundary $C$ and from (3.1) and (3.9) it follows that the coefficients in the $M \times N$ algebraic equations involve integrals of the form

$$
\int_{\mathbf{q}_{m}}^{\mathbf{q}_{m+1}} \Phi_{i j}\left(\mathbf{x}, \overline{\mathbf{q}}_{k}\right) d s(x), \quad \int_{\mathbf{q}_{m}}^{\mathbf{q}_{m+1}} \Gamma_{i j}\left(\mathbf{x}, \overline{\mathbf{q}}_{k}\right) d s(\mathbf{x}) .
$$

The first of the two integrals (3.10) presents no substantial difficulties and it may be readily integrated by employing the techniques outlined in Clements and Jones [2]. The second integral has a singularity in the integrand when $\overline{\mathbf{q}}_{k}$ lies on the segment $\left[\mathbf{q}_{m}, \mathbf{q}_{m+1}\right]$ (that is, when $m=k$ in (3.10)). However this integral may be readily evaluted as a Cauchy principal value integral and in certain important situations its value is zero. Likewise, if $\overline{\mathbf{q}}_{k}$ does not lie on the segment $\left[\mathbf{q}_{m}, \mathbf{q}_{m+1}\right]$ the integral may again be evaluated exactly, without recourse to approximate quadrature formulas, as we now show.

Let

$$
F_{\alpha j}(z, c)=\frac{1}{2 \pi} N_{\alpha m} \log (z-c) d_{m j}
$$

so that (3.2) and (3.3) may be written

$$
\begin{gathered}
\Phi_{i j}=\operatorname{Re}\left\{\sum_{\alpha} A_{i \alpha} F_{\alpha j}\left(z_{\alpha}, c_{\alpha}\right)\right\}, \\
\Gamma_{i j}=\operatorname{Re}\left\{\sum_{\alpha} L_{i p \alpha} F_{\alpha j}^{\prime}\left(z_{\alpha}, c_{\alpha}\right) n_{p}\right\},
\end{gathered}
$$

where the prime denotes differentiation with respect to $z_{\alpha}$. 
From (3.13)

$$
\int_{\mathbf{q}_{m}}^{\mathbf{q}_{m+1}} \Gamma_{i j}\left(\mathbf{x}, \mathbf{x}_{0}\right) d x(\mathbf{x})=\int_{\mathbf{q}_{m}}^{\mathbf{q}_{m+1}} \operatorname{Re}\left\{\sum_{\alpha}\left(L_{i 1 \alpha} n_{1}+L_{i 2 \alpha} n_{2}\right) F_{\alpha j}^{\prime}\left(z_{\alpha}, c_{\alpha}\right)\right\} d s(\mathbf{x}) .
$$

Now from (3.6) and (3.5)

and since

$$
L_{i 1 \alpha}=-\tau_{\alpha} L_{i 2 \alpha}
$$

$$
\left(n_{1}, n_{2}\right) d s=\left(d x_{2},-d x_{1}\right), \quad d z_{\alpha}=d x_{1}+\tau_{\alpha} d x_{2}
$$

it follows that the right hand side of (3.14) may be written in the form

$$
\begin{aligned}
& \operatorname{Re}\left\{\sum_{\alpha} L_{i 2 \alpha} \int_{\mathbf{q}_{m}}^{\mathbf{q}_{m+1}} F_{\alpha j}^{\prime}\left(z_{\alpha}, c_{\alpha}\right)\left(-\tau_{\alpha} n_{1}+n_{2}\right) d s(\mathbf{x})\right\} \\
&= \operatorname{Re}\left\{-\sum_{\alpha} L_{i 2 \alpha} \int_{z_{\alpha}^{m}}^{z_{\alpha}^{m+1}} F_{\alpha j}^{\prime}\left(z_{\alpha}, c_{\alpha}\right) d x_{\alpha}\right\}, \\
&=-\operatorname{Re}\left\{\sum_{\alpha} L_{i 2 \alpha} \int_{z_{\alpha}^{m}}^{z_{\alpha}^{m+1}} F_{\alpha j}^{\prime}\left(z_{\alpha}, c_{\alpha}\right) d z_{\alpha}\right\},
\end{aligned}
$$

where

$$
z_{\alpha}^{m}=x_{m}^{\prime}+\tau_{\alpha} y_{m}^{\prime} \text { with } \mathbf{q}_{m}=\left(x_{m}^{\prime}, y_{m}^{\prime}\right) \text {. }
$$

When $\mathbf{x}_{0}$ does not lie on the segment joining $\mathbf{q}_{m}$ to $\mathbf{q}_{m+1}$ it follows immediately from (3.11), (3.14) and (3.15) that

$$
\begin{aligned}
& \int_{\mathbf{q}_{m}}^{\mathbf{q}_{m+1}} \Gamma_{i j}\left(\mathbf{x}, \mathbf{x}_{0}\right) d x(\mathbf{x}) \\
& \quad=-\operatorname{Re} \frac{1}{2 \pi}\left\{\sum_{\alpha} L_{i 2 \alpha} N_{\alpha m}\left[\log \left(z_{\alpha}^{m+1}-c_{\alpha}\right)-\log \left(z_{\alpha}^{m}-c_{\alpha}\right)\right] d_{m j}\right\}
\end{aligned}
$$

while if $\mathbf{x}_{0}$ is on the line segment joining $\mathbf{q}_{m}$ to $\mathbf{q}_{m+1}$ then the integral in (3.15) is a Cauchy principal value integral and in this case it follows from (3.11), (3.14) and (3.15) that

$$
\begin{aligned}
\int_{\mathbf{q}_{m}}^{\mathbf{q}_{m+1}} & \Gamma_{i j}\left(\mathbf{x}, \mathbf{x}_{0}\right) d s(\mathbf{x}) \\
= & -\operatorname{Re} \frac{1}{2 \pi}\left[\sum_{\alpha} L_{i 2 \alpha} N_{\alpha m} \lim _{\varepsilon \rightarrow 0}\left\{\int_{z_{\alpha}^{m}}^{c_{\alpha}-\varepsilon} F_{\alpha j}^{\prime}\left(z_{\alpha}, c_{\alpha}\right) d z_{\alpha}+\int_{c_{\alpha}+\varepsilon}^{z_{\alpha}^{m+1}} F_{\alpha j}^{\prime}\left(z_{\alpha}, c_{\alpha}\right) d z_{\alpha}\right\}\right] \\
= & -\operatorname{Re} \frac{1}{2 \pi}\left[\sum _ { \alpha } L _ { i 2 \alpha } N _ { \alpha m } \left\{\log (-\varepsilon)-\log \left(z_{\alpha}^{m}-c_{\alpha}\right)\right.\right. \\
& \left.\left.+\log \left(z_{\alpha}^{m+1}-c_{\alpha}\right)-\log (\varepsilon)\right\}\right] d_{m j} \\
= & -\operatorname{Re} \frac{1}{2 \pi}\left[\sum_{\alpha} L_{i 2 \alpha} N_{\alpha m} \log \left|\frac{z_{\alpha}^{m+1}-c_{\alpha}}{z_{\alpha}^{m}-c_{\alpha}}\right|\right] d_{m j} \\
= & 0
\end{aligned}
$$


if $\mathbf{x}_{0}$ is equidistant from $\mathbf{q}_{m}$ and $\mathbf{q}_{m+1}$ and $\left[\mathbf{q}_{m}, \mathbf{q}_{m+1}\right]$ is a straight line segment.

It should be noted at this stage that this integral will be zero in many important cases since boundaries often consist of straight line segments and it is frequently practical to approximate curved boundaries by straight line segments.

For certain special cases of $(2.1)$ the integration of $\Gamma$ can be further simplified. For example in the case of Laplace's equation

$$
\frac{\partial^{2} \phi}{\partial x_{1}^{2}}+\frac{\partial^{2} \phi}{\partial x_{2}^{2}}=0
$$

the only nonzero constants are

$$
\begin{gathered}
\tau_{1}=i, \quad A_{11}=1, \quad L_{111}=1, \quad L_{121}=i, \\
N_{11}=1, \quad M_{11}=-i, \quad d_{11}=1,
\end{gathered}
$$

so that (3.16) and (3.17) provide

$$
\begin{aligned}
\int_{\mathbf{q}_{m}}^{\mathbf{q}_{m+1}} \Gamma\left(\mathbf{x}, \mathbf{x}_{0}\right) d x(\mathbf{x}) & =\frac{1}{2 \pi}\left[\arg \left(z_{1}^{m+1}-c_{1}\right)-\arg \left(z_{1}^{m}-c_{1}\right)\right] \\
& =\frac{\theta}{\pi} \quad \text { if } c_{1} \notin\left[z_{1}^{m}, z_{1}^{m+1}\right]
\end{aligned}
$$

and if $c_{1} \in\left[z_{1}^{m}, z_{1}^{m+1}\right]$

$$
\begin{aligned}
\int_{\mathbf{q}_{m}}^{\mathbf{q}_{m+1}} \Gamma\left(\mathbf{x}, \mathbf{x}_{0}\right) d x(\mathbf{x}) & =\operatorname{Re} \frac{1}{2 \pi}\left[i \log \left|\frac{z_{1}^{m+1}-c_{1}}{z_{1}^{m}-c_{1}}\right|\right] \\
& =0
\end{aligned}
$$

if $\overline{\mathbf{q}}_{m}$ is equidistant from $\mathbf{q}_{m}$ and $\mathbf{q}_{m+1}$. In (3.18), $\theta$ is the angle subtended at $\mathbf{x}_{0}$ by the integration segment $\left[\mathbf{q}_{m}, \mathbf{q}_{m+1}\right]$. This result is simple and exact, whereas the numerical integration methods usually employed can only give approximate values for the integrals. Note that (3.18) implies that for Laplace's equation the integral in question is zero if $x_{0}$ is on any extension of the straight line through $\mathbf{q}_{m}$ and $\mathbf{q}_{m+1}$.

In order to test the effectiveness of employing equation (3.1) in conjunction with (3.16) and (3.17) as against equation (3.8) some particular boundary value problems were considered. The procedures outlined in Clements and Jones [3] were used in obtaining a numerical solution from (3.8) while for (3.1) equations (3.16) and (3.17) were employed for the integral involving $\Gamma_{i j}$ and the procedures in [2] used for the integral involving $\Phi_{i j}$. The results obtained indicate that in general the two methods give numerical values which are in very close agreement and that there is little reason to prefer one method to the other on the basis of accuracy. Also in general the difference in computing times is negligible and not of any consequence. However in special cases when $\phi$ is given and constant along 
long segments of the boundary the method involving direct use of (3.1) can provide distinct advantages by offering the opportunity to employ long segments without any loss of accuracy. Finally, and perhaps most significantly, the method involving use of (3.1) facilitates considerable simplification in the construction of the computer program for the numerical solution of the integral equation.

\section{References}

[1] D. L. Clements, Boundary value problems governed by second order elliptic systems (Pitman, London, 1981).

[2] D. L. Clements and O. A. C. Jones, "The boundary integral equation method for the solution of a class of problems in anisotropic elasticity", J. Austral. Math. Soc. Ser. B 22 (1981), 394-407.

[3] D. L. Clements and F. J. Rizzo, "A method for the numerical solution of boundary value problems governed by second order elliptic systems", J. Inst. Math. Appl. 22 (1978), 197-202.

[4] M. A. Jaswon and G. T. Symm, Integral equation methods in potential theory and elastostatics (Academic Press, London, 1977). 